\title{
Evaluation of fingerprinting-based WiFi indoor localization coexisted with Bluetooth
}

\author{
Ling Pei ${ }^{1 *}$, Jingbin Liu², Yuwei Chen ${ }^{3}$, Ruizhi Chen² and Liang Chen²
}

\begin{abstract}
WiFi and Bluetooth are two most commonly used short range wireless communication technologies. Recent years, with increasing number of WiFi and Bluetooth mobile terminals, tags, and other devices, a demand for integration and coexistence of these two technologies including their positioning function is booming. In this paper, we firstly investigate the interferences between WiFi and Bluetooth signals from the signal and protocol perspectives. Secondly, the principle of fingerprinting approach for WiFi positioning is introduced. In order to evaluate the performance of WiFi fingerprinting coexisted with Bluetooth, both occurrence-based and Weibull-based approaches are utilized for generating the database. Field tests present the interference in the WiFi and Bluetooth coexistence environments. A WiFi mobile device with a Bluetooth device nearby obtains poor positioning results due to the interference. Weibull-based database has more robust performance than occurrence-based database in the coexistence environments.
\end{abstract}

Keywords: WiFi, Bluetooth, Coexistence, Positioning, Fingerprinting

\section{Introduction}

In the fast growing mobile device market, the location capability has become one of the most demanded features. To address the location capability, an increasing number of research focus on positioning and navigation technologies for both outdoor and indoor environments. The Global Navigation Satellites Systems (GNSS) greatly enrich the end users' outdoor activities by mobile devices. However, the degraded areas e.g. indoors and urban canyon are still challenges for satellite based positioning technologies because of well known problems such as the weak signal or non line-of-sight (NLOS) conditions between mobile users and satellites.

In order to solve the problem of positioning and navigation in GNSS-degraded or denied areas, diverse technologies are broadly researched (Kraemer and Eisfeller, 2009). Most research topics focus on high-

\footnotetext{
* Correspondence: ling.pei@sjtu.edu.cn

${ }^{1}$ Shanghai Key Laboratory of Navigation and Location-based Services, School of Electronic Information and Electrical Engineering, Shanghai Jiao Tong University, Shanghai, China

Full list of author information is available at the end of the article
}

sensitivity GNSS (Syrjärinne, 2001), optical navigation systems (Mulloni et al., 2009, Rouzaud and Skaloud, 2011, Zhou et al., 2015), acoustic solutions (Priyantha et al., 2000, Jiang et al., 2015)0, WiFi (Bahl and Padmanabhan, 2000), Bluetooth (Pei et al., 2010a, Pei et al., 2010b, Pei et al., 2010c, Chen et al., 2011a, Chen et al., 2012a, Chen et al., 2013), ZigBee (Sugano et al., 2006), Ultra Wide Band (Pahlavan et al., 2006), cellular networks (Syrjärinne, 2001), RFID (Hightower et al., 2000), magnetic localization (Storms, 2010), inertial measurement units (Foxlin, 2005 and Chen et al., 2010), signals of opportunity (Mathews et al., 2011, Chen et al., 2012b, Pei et al., 2016), dead reckoning approaches (Pei et al., 2011c, Chen et al., 2011c, Pei et al., 2012a, Pei et al., 2013, Qian et al., 2015a, Qian et al., 2015b), and also hybrid solutions (Chen et al., 2009, Pei et al., 2009, Pahlavan et al., 2010, Liu et al., 2010, Chen et al., 2011b, Kuusniemi et al., 2012, and Liu 2012a, Liu et al., 2012b).

RF-based technologies, such as WiFi, Bluetooth, cellular network, and RFID, are the highest potential alternatives to positioning in degraded environments because of the existing RF infrastructures. The first wellknown WiFi-based positioning system, namely RADAR 
(Bahl and Padmanabhan, 2000), utilizes kNN positioning algorithm to compute the location of a mobile device based on radio signal strength (RSS) from visible access points (APs) nearby. Using WiFi RSSIs, Ekahau (Ekahau Inc, 2017) provides an easy and cost-effective solution for locating people, assets, inventory and other objects. Similar to the Ekahau system based on specific RF tags, the Active Badge (Want et al., 1992) system uses ceiling-mounted infrared sensor detectors to detect signals from a mobile active badge. Place Lab (Schilit et al., 2003) combines Wi-Fi, GSM and Bluetooth devices as global beacons. Chen et al. (Chen et al., 2009) proposes a multi-network approach to improve the availability and reliability of navigation and positioning. However, the deployment of diverse wireless devices operating in the $2.4 \mathrm{GHz}$ unlicensed band, is met with growing concerns about signal interference and performance degradation. Pei et al., (2012b) present the preliminary results of WiFi positioning with Bluetooth interferences. In this paper, we will evaluate the performance of fingerprinting-based WiFi positioning in Bluetooth and WiFi coexistence environments. Instead of using the conventional occurrence-based database, we propose the Weibullbased fingerprint database which has more robust performance when the WiFi spectrum is affected by coexisted Bluetooth signals.

We organize this paper as follows: first of all, we will investigate the causes of interference from $\mathrm{WiFi}$ and Bluetooth protocol perspective in Section 2. Then, we Then, we present two types of fingerprint databases for WiFi positioning in Section 3. Section 4 describes the algorithms for positioning based on the fingerprint database. In Section 5, we conduct several experiments to evaluate the WiFi positioning performance in Bluetooth rich environments. Finally, concluding remarks and discussions are given in Section 6.

\section{Interference analysis}

Interferences within $2.4 \mathrm{GHz}$ band bring growing concerns in wireless communication society. The interference not only exists within the same wireless technology but also among different signal sources sharing the same band. We will discuss the interferences related to WiFi and Bluetooth in this section.

\subsection{WiFi}

The IEEE 802.11 family e.g. IEEE $802.11 \mathrm{~b}$ and $802.11 \mathrm{~g} / \mathrm{n}$ protocols are the most widely used wireless local area network technologies nowadays. They operate in the industrial, scientific and medical (ISM) radio bands of $2.4 \mathrm{GHz}$. $802.11 \mathrm{~b}$ and $802.11 \mathrm{~g} / \mathrm{n}$ control their interference and susceptibility to interference by using direct-sequence spread spectrum (DSSS) and orthogonal frequency-division multiplexing (OFDM) signaling methods, respectively. The 2.4 GHz band is divided into 14 channels spaced $5 \mathrm{MHz}$ apart, beginning with channel 1 , which is centered on $2.412 \mathrm{GHz}$. The bandwidth of each channel is $22 \mathrm{MHz}$ as shown in Fig. 2. It means that a channel will be interfered by two nearest channels from either side. With the increasing distribution density of WiFi devices in a limited space, interference occurs because of the channels overlapping. The top image of Fig. 1 shows the interference from the overlapped channels of $802.11 \mathrm{~b}$. Compared to the case shown in the top image, the interference is reduced significantly in the bottom image of Fig. 1 since we allocate $802.11 \mathrm{~b}$ WiFi APs' channels at No. 1, 6, and 11, and $802.11 \mathrm{~g} / \mathrm{n} \mathrm{WiFi}$ APs' channels at No. 1 and 13. Therefore, to improve the throughput of WiFi communication in a target area, we recommend assigning an AP's channel at No. $1,6,11$, or 14 for $802.11 \mathrm{~b}$ and No. $1,5,9$, or 13 for $802.11 \mathrm{~g} / \mathrm{n}$, respectively, if the number of visible APs in the area are less than five. Otherwise, the interference is unavoidable.

\section{Bluetooth}

Bluetooth, a technology with low power consumption for short-range wireless data and voice communication, also works in the $2.4 \mathrm{GHz}$ ISM band and share spectrum with WiFi. Bluetooth has been utilized in the communication and proximity market for a long time. As widely supported by mobile devices, Bluetooth is a potential technology to become an alternate for indoor positioning. The effective range of the radio signal of a standard Class 1 Bluetooth device is up to $100 \mathrm{~m}$, while that for the Class 2 device is about $20-30 \mathrm{~m}$ according to the specifications of Bluetooth 2.0.

Bluetooth and WiFi both operate in the $2.4 \mathrm{GHz}$ ISM band and share spectrum, as shown in Fig. 2. These technologies will often be located in close physical proximity to another and sometimes these two types of networks may even overlap each other. Figure 3 gives a scenario of Bluetooth interfering with a WiFi device. A possible way whereby Bluetooth devices are able to avoid interference from other wireless devices is through a technique known as spreadspectrum frequency hopping. By using the "hopping" method, a device will use one of 79 different and randomly chosen frequencies within an assigned range. The device frequently changes frequencies from one to another one with 1600 times per second in a connected state and 3200 times per second in an inquiring state. As a result, more devices can use a portion of the radio spectrum. The risk of a device like WiFienabled device interfering with Bluetooth devices is minimized, since any interference on a specific frequency will last for only a fraction of a second. 

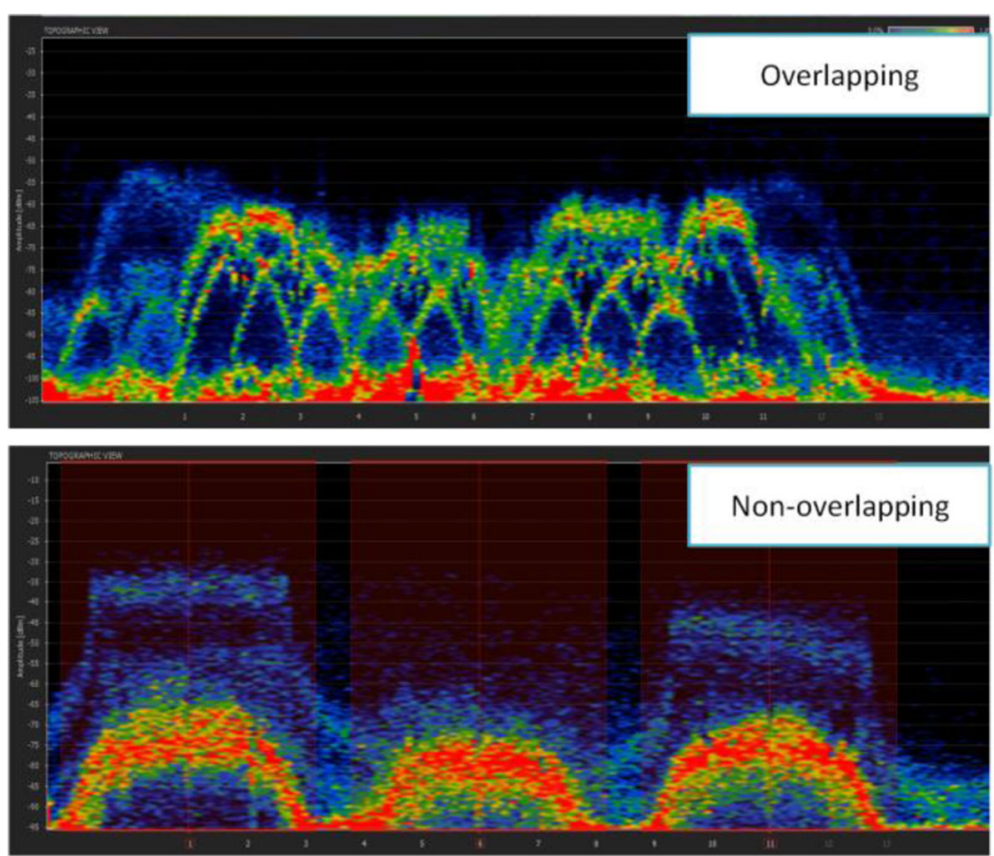

Fig. 1 Frequency spectrum of WiFi channel allocations

However, on the other hand, WiFi still suffers from the hopping interference of Bluetooth as shown in Fig. 3. The spectrum with narrow bandwidth is from Bluetooth and the spectrum with wider bandwidth is WiFi signal centered at channel 11 . The WiFi spectrum is corrupted by the Bluetooth which keeps hopping from channel 1 to 79 .

Bluetooth version 1.2 and afterwards enable an enhanced technology called Adaptive Frequency Hopping
(AFH) to mitigate the interference in a coexistence environment. AFH allows Bluetooth devices to measure the quality of the wireless signal and then determine if there are bad channels present on specific frequencies due to interference from other wireless devices. As shown in Figs. 1 and 4, if bad channels are present on a specific frequency, the Bluetooth device will adjust its hopping sequence to avoid them. As a result, the Bluetooth connection is stronger, faster, and more reliable.

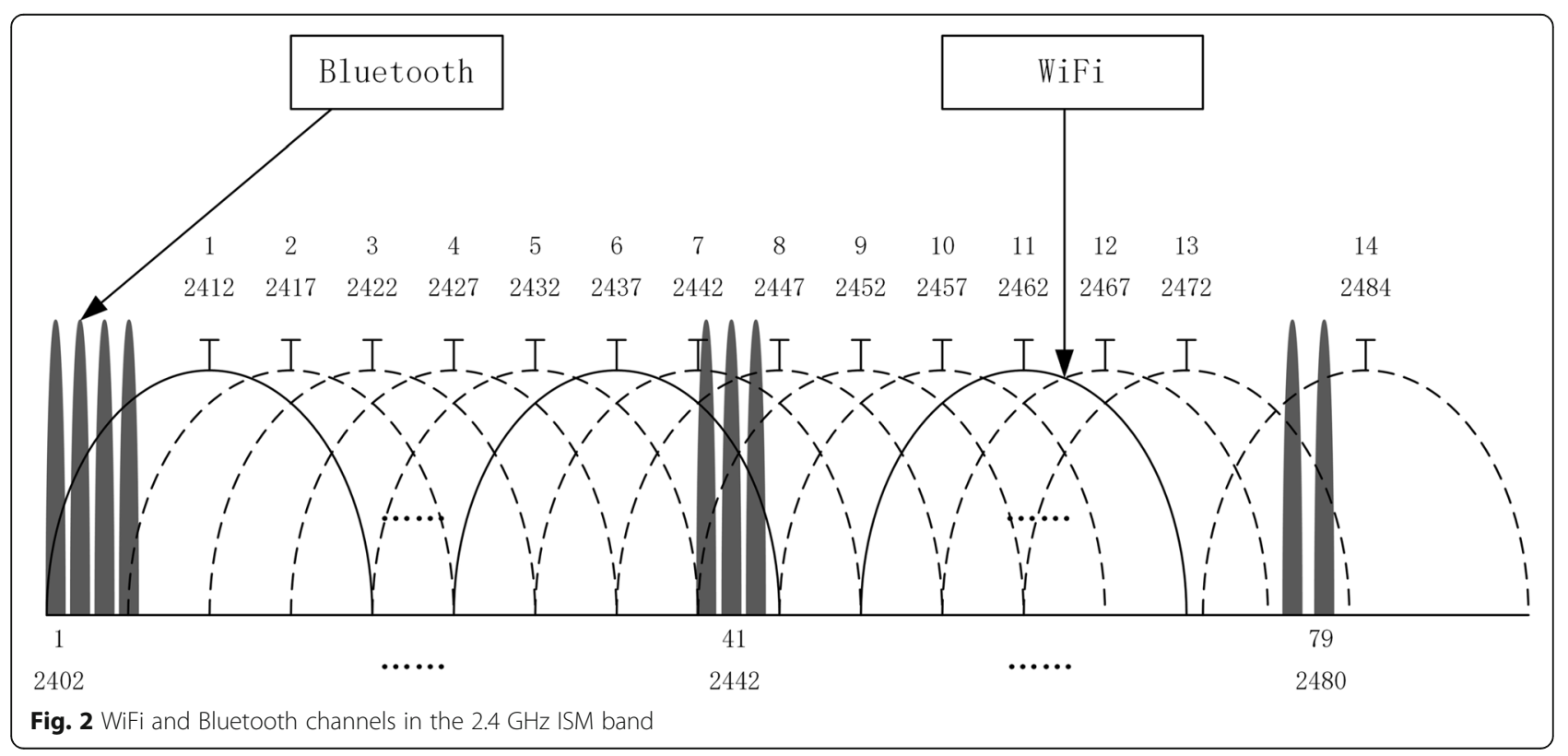




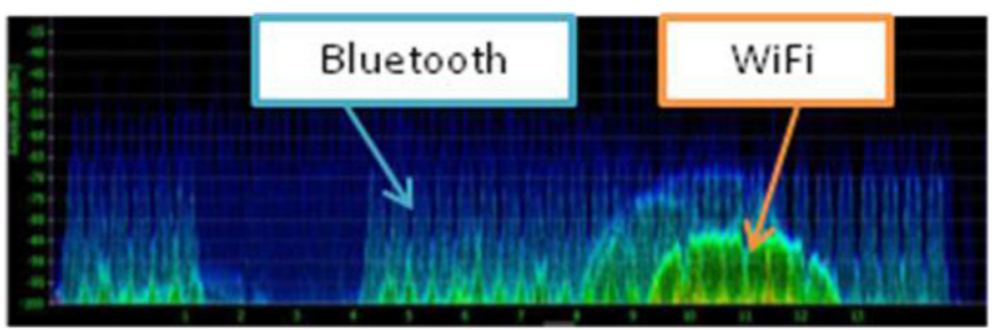

Fig. 3 A scenario of Bluetooth interfering with a WiFi device

Meanwhile, the throughput of WiFi networks is also improved without Bluetooth interfering. Unfortunately, the up-to-date version of AFH can merely support Bluetooth communication with an established connection.

Therefore, in Bluetooth rich environments, WiFi signals might be interfered by nearby Bluetooth devices. In consequence, the interference will effect on both fingerprinting generation and positioning results of a fingerprinting-based WiFi positioning solution. During the phase of generating a fingerprint database, the inband interference e.g. Bluetooth signals disturb the WiFi RSSI readings, which may distort the signal distributions in the fingerprint database and decrease the positioning accuracy.

The WiFi access points might be missed by a mobile device because the mobile device does not capture the signals broadcasted by the access points during a scanning cycle defined by the WiFi chipset in the mobile device. With the increasing number of APs in range or coexisted with the devices working at the similar frequency as WiFi, the cases of missing WiFi APs will increase. In this paper, we denote the miss-scan rated as a term to evaluate the missing times during a specific interval of WiFi scanning.

\section{Methods}

To maximize the reliability of WiFi positioning in complex signal environments, the fingerprinting algorithm must be carefully designed to discover a situation in

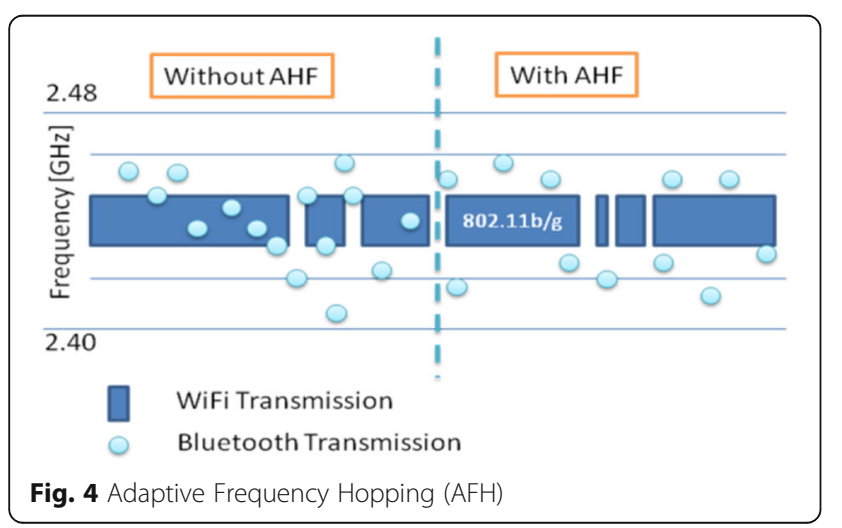

which some of the received signal strength measurements may be miss-scanned because of the prevailing interference. In this paper, occurrence-based fingerprint database and Weibull-based fingerprint database are implemented.

\section{Occurrence-based fingerprint database}

The most common observation in WiFi positioning solutions is RSSI, which is an indication of a power level received by an antenna. In principle, the RSSI observation can refer to the distance between a WiFi access point and a WiFi terminal. This distance-RSSI relationship can be further used for triangulating a terminal's position on the condition that the terminal obtains RSSIs from at least three different access points. In practical, however, the interference and non-line-of-sight (NLOS) make the distance-RSSI relationship hard to precisely model. Instead of using the triangulation solution based on distance-RSSI relationship directly, the fingerprinting is more a popular approach within a specific space.

During the phase of generating a WiFi fingerprint database, a targeted area is divided into a number of grids. The center of each grid is considered as a reference point. The coordinates of the reference points $\left(\mathrm{x}_{\mathrm{n}}, \mathrm{y}_{\mathrm{n}}\right)$ are determined in advance. The RSSI measurements at each reference point from all "visible" WiFi APs are collected and stored as fingerprints in the database. At each reference point, the RSSI probability distributions of all WiFi APs are stored.

At each reference point, the RSSI probability distributions of all APs are stored. If we denote the fingerprint for the $i$-th reference point as $R_{i}$, then, we have

$$
R_{i}=\left[\begin{array}{cccc}
P\left(A_{1} O_{1} \mid R_{i}\right) & P\left(A_{2} O_{1} \mid R_{i}\right) & \cdots & P\left(A_{k} O_{1} \mid R_{i}\right) \\
P\left(A_{1} O_{2} \mid R_{i}\right) & P\left(A_{2} O_{2} \mid R_{i}\right) & \cdots & P\left(A_{k} O_{2} \mid R_{i}\right) \\
\vdots & \vdots & \ddots & \vdots \\
P\left(A_{1} O_{v} \mid R_{i}\right) & P\left(A_{2} O_{v} \mid R_{i}\right) & \cdots & P\left(A_{k} O_{v} \mid R_{i}\right)
\end{array}\right]
$$

where $A$ stands for the AP, while Orefers to the RSSI observation. 
In the occurrence-based fingerprinting approach, the probability of a RSSI measurement $O_{n}$ which is collected from the AP $A_{m}$ at the reference point $R_{i}$ can be expressed as (Pei et al., 2010b)

$$
P\left(A_{m} O_{n} \mid R_{i}\right)=\frac{C_{O_{n}}}{N_{i}}
$$

where $C_{O_{n}}$ is the number of occurrences that the RSSI observation $O_{n}$ appeared in the training data set of the $i$-th reference point. Here $N_{i}$ is the total number of training samples collected at the $i$-th reference point. The entire fingerprint database is expressed as

$$
\mathrm{D}=\left[R_{1}, R_{2}, \ldots, R_{w}\right]
$$

where $W$ is the maximum number of the reference points in the radio map.

To speed up the computation process, a bin-based solution is adopted. The signal strength distribution is divided into $\mathrm{p}$ bins. The fingerprints for the $i$-th reference point can be redefined as

$R_{i}=\left[\begin{array}{cccc}P\left(A_{1} B_{1} \mid R_{i}\right) & P\left(A_{2} B_{1} \mid R_{i}\right) & \cdots & P\left(A_{k} B_{1} \mid R_{i}\right) \\ P\left(A_{1} B_{2} \mid R_{i}\right) & P\left(A_{2} B_{2} \mid R_{i}\right) & \cdots & P\left(A_{k} B_{2} \mid R_{i}\right) \\ \vdots & \vdots & \ddots & \vdots \\ P\left(A_{1} B_{p} \mid R_{i}\right) & P\left(A_{2} B_{p} \mid R_{i}\right) & \cdots & P\left(A_{k} B_{p} \mid R_{i}\right)\end{array}\right]$

In the occurrence-based database, at the $i$-th reference point, the probability of the RSSI measurements within the bin $B_{n}$ for AP $A_{m}$ can be expressed as

$P\left(A_{m} B_{n} \mid R_{i}\right)=\sum_{j=E_{n-1}}^{j<E_{n}} \frac{C_{O_{j}}}{N_{i}}$

where $E_{n-1}$ and $E_{n}$ are the left and right edges of bin $B_{n}$ respectively. $C_{O_{j}}$ stands for the number of occurrences that the value of the RSSI measurement appeared within the range of $\left[E_{n-1}, E_{n}\right)$. All the RSSI measurements in the bin $B_{n}$ are cumulated for counting the occurrence probability.

\section{Weibull-based fingerprint database}

As the example shown in Fig. 3, WiFi RSSI readings might be decayed or jammed by the interference signals. We therefore might obtain fewer samples to estimate of the RSSI probability distribution in the bin-based solution than the case without interference. In this paper, we introduce the Weibull function to proximate the RSSI probability distribution. The Weibull function is a classical method for modeling the signal strength of radio propagation (Sagias \& Karagiannidis, 2005). The Weibull probability density function can be expressed as

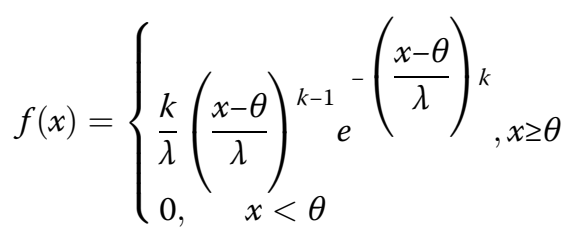

The cumulate distribution function is defined as

$$
F(x)=1-e^{-\left(\frac{x-\theta}{\lambda}\right)^{k}}
$$

where $x$ is the variable of the function, $k$ is the shape parameter, $\lambda$ is the scale parameter, and $\theta$ is the shift parameter. When $\theta=0$, this reduces to a 2-parameter distribution.

The parameters of the Weibull function can be estimated with a limited number of RSSI sample measurements. The function parameters $(\lambda, k, \theta)$ can be calculated with (Papoulis, 2002):

$$
\begin{aligned}
& k=\delta / \ln (2), \quad 1.5 \leq k \leq 2.5 \\
& \lambda=\left\{\begin{array}{cc}
2 \times(k+0.15) & \delta<2 \\
\delta \times(k+0.15) & 2 \leq \delta \leq 3.5 \\
3.5 \times(k+0.15) & \delta>3.5
\end{array}\right.
\end{aligned}
$$

$$
\theta=\bar{O}-\lambda \times \Gamma(1+1 / k)
$$

$$
\bar{O}=\frac{1}{n} \sum_{i=0}^{n} O_{i}
$$

$$
\delta=\sqrt{\frac{1}{n} \sum_{i=0}^{n}\left(O_{i}-\bar{O}\right)^{2}}
$$

where $\bar{O}$ is the mean value of the RSSI observation set $O_{i}$, $\delta$ is the standard deviation. $\Gamma$ is the gamma function. The term $(k+0.15)$ is an approximation of the expression $1 / \sqrt{\Gamma\left({ }^{\circ} 1+2 / k\right)-\Gamma^{2}(1+1 / k)}$ when $1.5 \leq k \leq 2.5$.

Because the RSSI observations are rounded to an integer, for each possible RSSI observation in this study, the distribution probability can be expressed as

$$
P(x)=F(x+0.5)-F(x-0.5)
$$

The probability for each bin in the fingerprint database can be generated as 


$$
P\left(A_{m} B_{n} \mid R_{i}\right)=\int_{x}^{x+w} f(x) d x=F(x+w)-F(x)
$$

where $W$ is the width of the bin, $x$ is the RSSI value at the left edge of bin.

\section{Fingerprinting-based WiFi localization}

Based on the constructed fingerprint database using the algorithms described in Section 3, the positioning phase determines the current location based on the obtained RSSI observations in real time. The Bayesian theorem and the Histogram Maximum Likelihood algorithm are used for position estimation.

Given the RSSI measurement vector $\vec{O}=\left\{O_{1}, O_{2} \ldots O_{k}\right\}$ from APs, the problem is to find the location $l$ with the conditional probability $P(l \mid \vec{O})$ being maximized. Using the Bayesian theorem (Pei et al., 2010b)

$$
\operatorname{argmax}_{l}[P(l \mid \vec{O})]=\operatorname{argmax}_{l}\left[\frac{P(\vec{O} \mid l) P(l)}{P(\vec{O})}\right]
$$

where $P(\vec{O})$ is constant for all $l$, therefore, the Eq. (15) can be reduced as

$$
\operatorname{argmax}_{l}[P(l \mid \vec{O})]=\operatorname{argmax}_{l}[P(\vec{O} \mid l) P(l)]
$$

We assume that the mobile device has equal probability to access each reference point, so $P(l)$ can be considered as constant in this case, Eq. (17) can be simplified as

$$
\operatorname{argmax}_{l}[P(l \mid \vec{O})]=\operatorname{argmax}_{l}[P(\vec{O} \mid l)]
$$

Now it becomes a problem of finding the maximum conditional probability of

$$
P(\vec{O} \mid l)=\prod_{n=1}^{k} P\left(O_{n} \mid l\right)
$$

where the conditional probability $P\left(O_{n} \mid l\right)$ is derived from the RSSI distribution pre-stored in the fingerprint database.

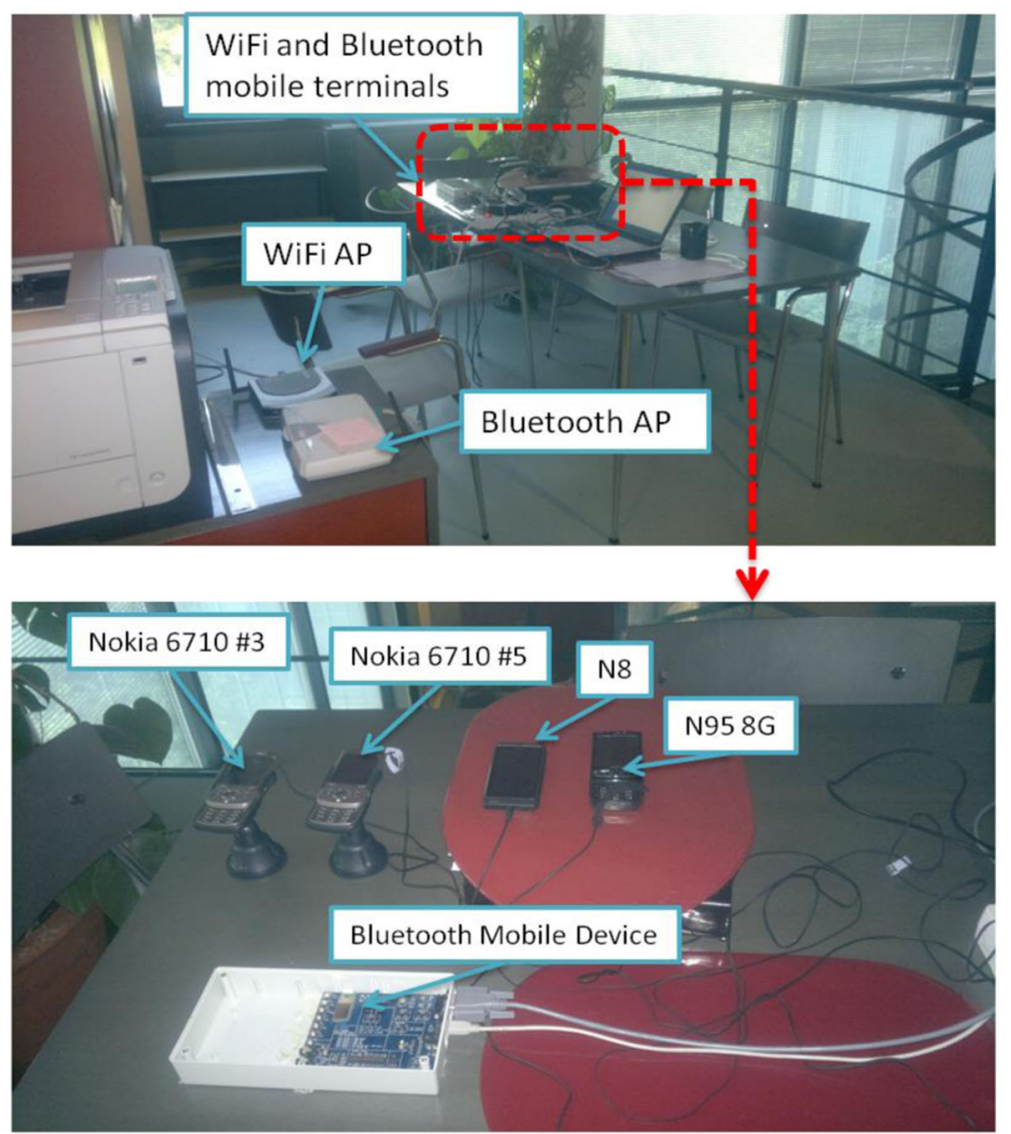

Fig. 5 The test scene in FGl 


\section{Results}

To evaluate the performance of $\mathrm{WiFi}$ positioning in a coexistence environment, we conducted a series of tests.

\section{Test setups}

All tests were performed in an office building with 16 WiFi APs and 13 Bluetooth APs existed. We set the neighboring WiFi APs at channel 1, 6, 11 respectively to avoid the interference from WiFi. And all the RSSs of an AP are collected from the same channel set beforehand.

Figure 5 shows the test scene where the test mobile devices include four smart phones and one Bluetooth mobile terminal standing on a table. About three meters apart from the mobile devices, there were one WiFi AP and Bluetooth AP standing on another table. In order to eliminate the influence from the difference of mobile devices, we selected four smart phones. Two of them were a same model. We developed an application which was continuously scanning nearby WiFi APs and logging the RSSI observations on each smart phone. Meanwhile, another application was developed in the firmware of the Bluetooth mobile device to discover the Bluetooth APs in range.

\section{Test scenarios}

In order to investigate the following facts in the coexistence environment:

- RSSI vs. miss-scanned rate,

- Bluetooth radio impacts on WiFi RSSI observations,

- Bluetooth radio impacts on WiFi positioning,

- The interference difference between AP side and terminal side,

- Temporal impacts on WiFi RSSI observations,

- AFH vs. non-AFH, we designed the following test scenarios

- Case 1: WiFi coexistence performance test with Bluetooth AP on and Bluetooth mobile device on.

- Case 2: WiFi coexistence performance test with Bluetooth AP on and Bluetooth mobile device off.

- Case 3: WiFi standalone performance test with Bluetooth AP off and Bluetooth mobile device off.

- Case 4: WiFi coexistence performance test with Bluetooth mobile device AFH capability on and off.

\section{Test results}

The tests lasted 4 days. The minimum test duration was 6 h. The RSSI observations collected from 16 WiFi APs, one example as shown in Fig. 6, indicate that the missscanned rate of WiFi RSSI observations increases with the RSSI value decreasing. The value of zero here denotes that the signal strength of a WiFi AP is so weak that a smart phone is not able to discover. Miss-scanned rate shows the fraction of scans where no APs were detected.

Figure 7 indicates that the miss-scanned rate of WiFi RSSI observations is higher in the coexistence environment than in the standalone environment. Bluetooth APs have less impact on the WiFi missscanned rate than the Bluetooth mobile device. The miss-scanned rates reach 1 with $\mathrm{WiFi}$ AP 4, 5, 7, 8, $9,12,13,14,15$, and 16, which means that these APs are too weak to be detected by the mobile device. If there is a Bluetooth mobile device nearby, the missscanned rate recorded by a WiFi mobile device goes higher, which is proved by AP $1,2,3,6,10$, and 11 . In other word, the visibilities of all the WiFi APs in range are decreased because the nearby Bluetooth mobile device effected on the scanning procedure

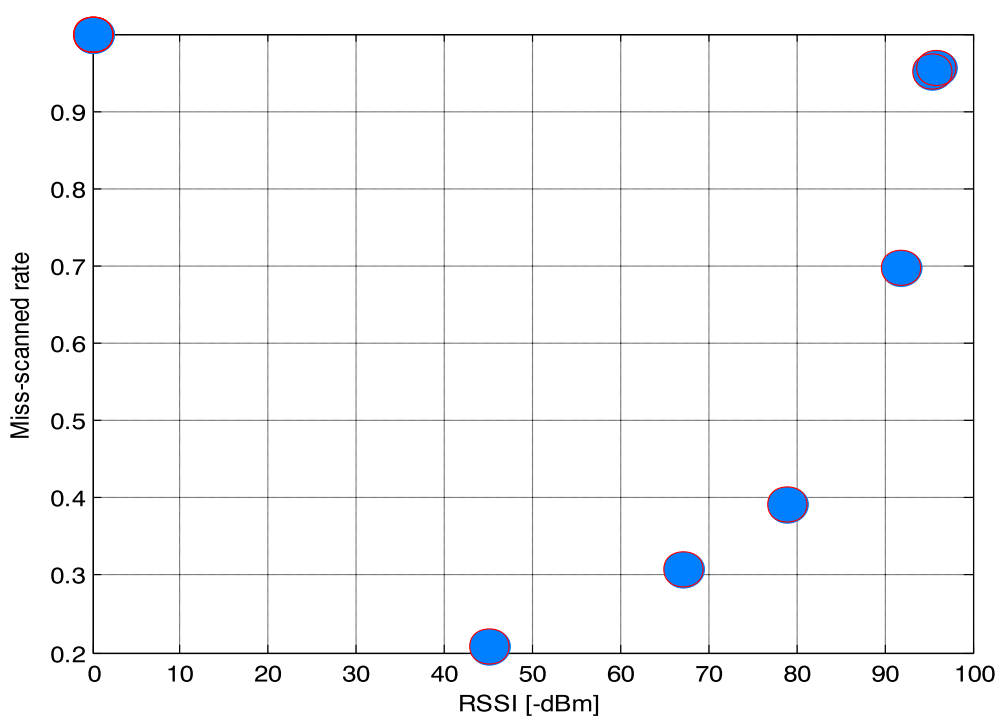

Fig. 6 RSSI vs. miss-scanned rate 


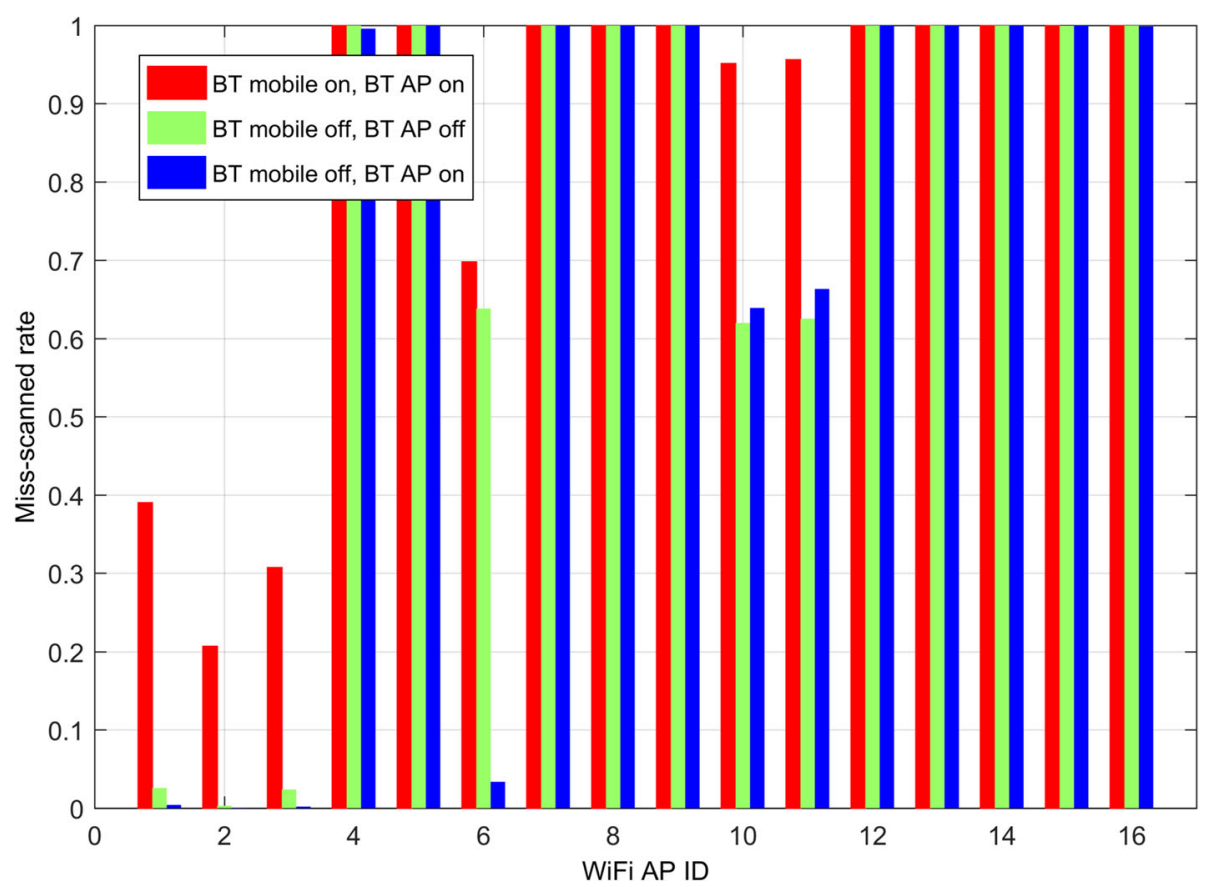

Fig. 7 WiFi standalone performance vs. coexistence performance (BT: Bluetooth)

executed on the WiFi mobile device. Bluetooth APs give less interference on WiFi scanning if the Bluetooth APs are far away from the WiFi mobile device. Otherwise the Bluetooth AP will impact on the WiFi mobile device as the nearby mobile device does.

WiFi RSSI observations might vary by the time changing. Therefore, we tested on the same smart phones in the same test environment with Bluetooth APs and the Bluetooth mobile device power off. Test 1 lasted $36 \mathrm{~h}$, and test 2 lasted $12 \mathrm{~h}$. Since test 2 was executed during the working hours, interference such as personal Bluetooth-enabled devices might be introduced. As shown in Table 1, two tests have the similar mean values. Test 2 , however, has slightly higher standard deviation and miss-scanned rate. In general, the performances of two tests are very close. Table 2 shows a certain difference of RSSI observations among the different models of smart phones.

Table 1 Day to day comparison of WiFi RSSI observations

\begin{tabular}{lllll}
\hline WiFi AP ID & & 1 & 2 & 3 \\
\hline Mean $(-\mathrm{dBm})$ & Test 1 $(36 \mathrm{~h})$ & 76.32 & 46.03 & 67.08 \\
& Test 2 (12 h) & 74.81 & 45.33 & 68.47 \\
Standard deviation & Test 1 (36 h) & 0.54 & 0.52 & 0.65 \\
(dBm) & Test 2 (12 h) & 0.89 & 0.72 & 1.16 \\
Miss-scanned rate & Test 1 (36 h) & 0.03 & 0.003 & 0.02 \\
& Test 2 (12 h) & 0.07 & 0.006 & 0.03 \\
\hline
\end{tabular}

Considering that many Bluetooth devices implement the Adaptive Frequency Hopping (AFH) mechanism since Bluetooth V1.2 released, we also tested the performance of WiFi RSSI sampling under the circumstances of both non-AFH and AFH mechanisms. We enabled the Bluetooth AFH capability on a Bluetooth mobile device and kept the communication with a Bluetooth AP. As shown in Fig. 8, compared with the non-AFH results, the WiFi RSSI sampling performance is improved in the AFH-enabled test case such as AP 12 , 3, and 6, where the RSSI values are higher than $-80 \mathrm{dBm}$. However, the AFH does not help if the RSSI value is too low to detect. The AFH-enable Bluetooth might decrease the WiFi throughout capability in the case that WiFi signals are very weak but

Table 2 Devices comparison of WiFi RSSI observations

\begin{tabular}{lllll}
\hline WiFi AP ID & & 1 & 2 & 3 \\
\hline Mean (-dBm) & N8 & 79.58 & 45.00 & 67.66 \\
& N95 8G & 78.82 & 37.07 & 66.27 \\
& $6710-5$ & 76.32 & 46.03 & 67.08 \\
Standard deviation & N8 & 0.86 & 0.07 & 0.76 \\
$(\mathrm{dBm})$ & N95 8G & 0.51 & 0.38 & 0.92 \\
& $6710-5$ & 0.54 & 0.52 & 0.65 \\
Miss-scanned rate & N8 & 0.004 & 0.0005 & 0.002 \\
& N95 8G & 0.007 & 0.0003 & 0.003 \\
& $6710-5$ & 0.03 & 0.003 & 0.02 \\
\hline
\end{tabular}




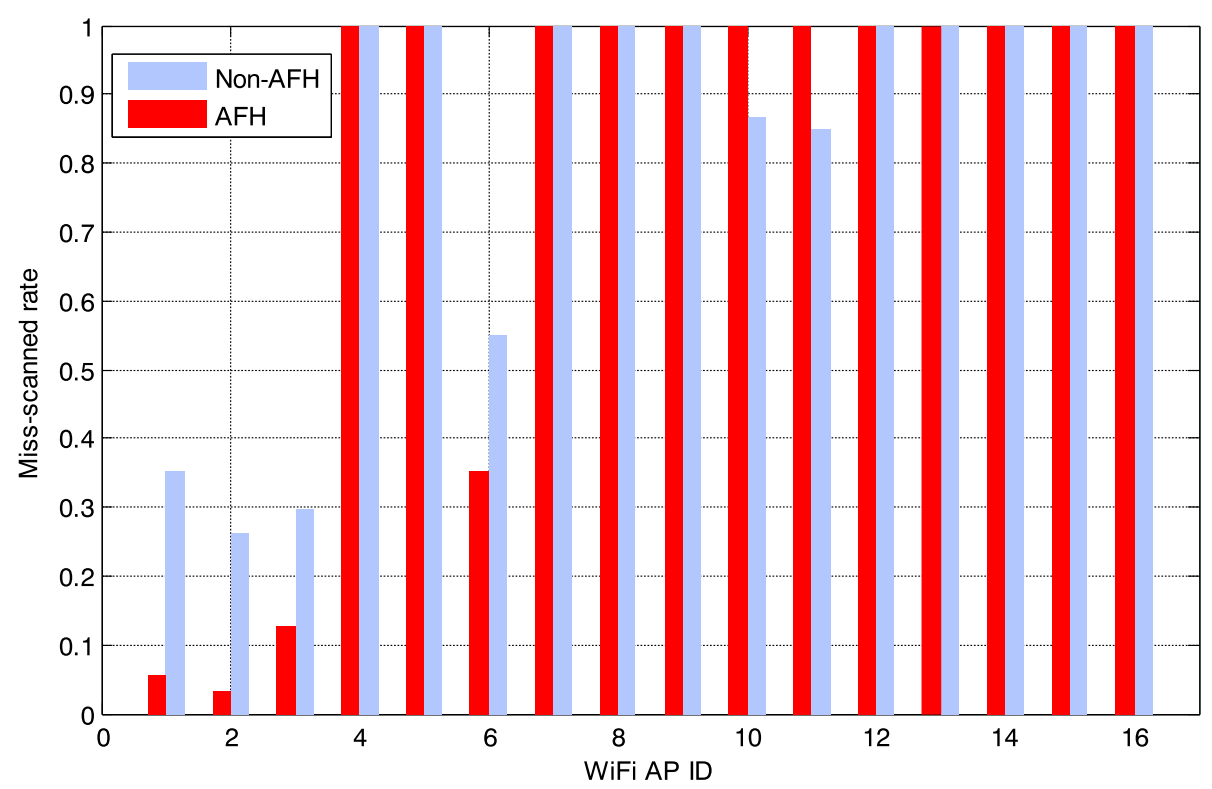

Fig. 8 Non-AFH vs. AFH

still visible occasionally. We suggest that the WiFi fingerprints only utilize the high RSSIs and avoid the unstable RSSI measurements while WiFi signals are low.

An indoor test was carried out in a corridor on the third floor at the Finnish Geodetic Institute as shown in Fig. 9. Thirteen Bluetooth APs were deployed in the whole building, with 5 APs on the second floor and the others are on the third floor. 13 of WiFi APs were deployed at the same location with the Bluetooth APs. The other 3 WiFi APs were located at the first floor. The Bluetooth mobile device with a self-developed application is applied to control the Bluetooth module to scan the APs nearby, collect the RSS from the detected APs and send the measurements to the laptop via a serial port. The sampling interval could be adjusted within 4-11.25 s according to the scanning priority chosen. We set $4 \mathrm{~s}$ in the field tests in this paper. A Google Nexus mobile phone was used as target device for WiFi positioning tests. The phone was placed at 32 reference points marked at the third floor.

Table 3 gives WiFi positioning results in standalone and coexistence environments provided with occurrence-based and Weibull-based fingerprint database. In the case of Bluetooth APs and the Bluetooth mobile device are all powered on, WiFi positioning accuracy has the worst performance. While in the case that all Bluetooth devices are powered off, we achieve the best positioning accuracy. Weibullbased fingerprint database gives a better performance than occurrence-based fingerprint database in the coexistence environment. WiFi positioning results significantly decrease if a Bluetooth mobile device is working nearby, even though the Weibull-based fingerprint database is applied.

\section{Conclusion and discussion}

The location-based service market using WiFi and Bluetooth devices is growing rapidly. Meanwhile, the number of WiFi and Bluetooth terminals, tags, and other devices are increasing in a pervasive way. Those drive an increasing demand for integration and coexistence of these two technologies. This paper gives the preliminary results of $\mathrm{WiFi}$ positioning in a WiFi and Bluetooth coexistence environment.

The results indicate that stronger RSSI values usually result in more stable RSSI observations. The closer a Bluetooth device is, the lower accuracy of WiFi positioning might be caused. WiFi positioning suffers from the interference in a coexistence environment. Weibullbased fingerprint database has more robust performance than occurrence-based fingerprint database. However, the Weibull-based approach can only relieve the effects of RSSI observation miss-scanned. Weibull-based approach cannot solve the problem that the interferences distort the RSSI observations. RSSI observations vary since the differences exist in WiFi devices. Normalization is recommended if the WiFi module of a positioning device is different from the one used for generating the fingerprint database. AFH mechanism decreases the Bluetooth interference to WiFi RSSI scanning. However, AFH mechanism can only be deployed in the Bluetooth connection state. Bluetooth devices in an inquiring state still interfere with WiFi RSSI scanning. Miss-scanned samples will lower the WiFi positioning performance if those samples are considered as a feature of the RSSI fingerprinting. Furthermore, miss- 


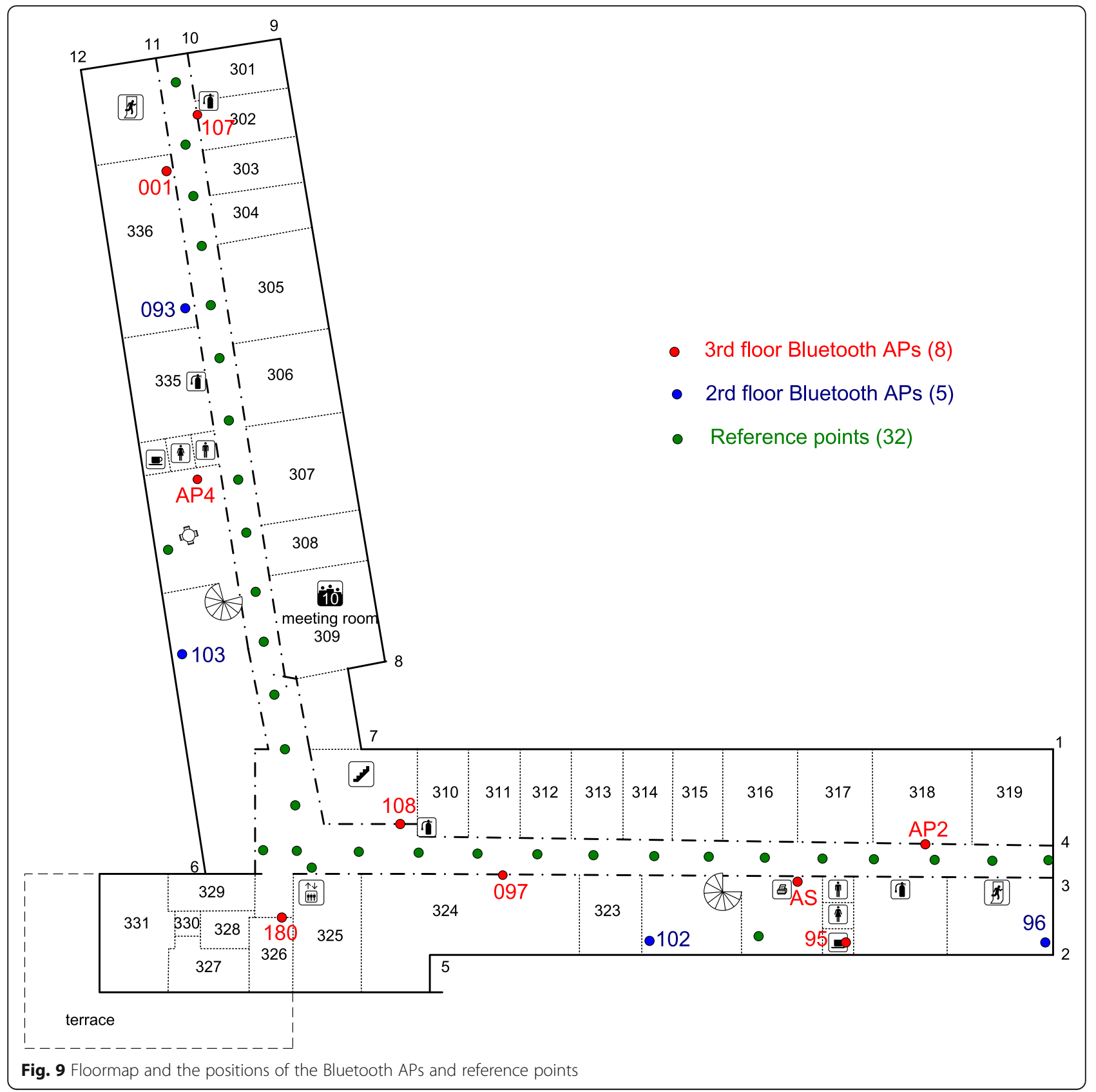

Table 3 WiFi positioning performance (unit: $m$ )

\begin{tabular}{lllllllll}
\hline BTAP & WiFi AP & WiFi Phone & BT Mobile & Database Type & Mean Err & RMSE & Max Err & Min Err \\
\hline OFF & ON & ON & OFF & Weibull & 2.33 & 4.58 & 17.12 & 0 \\
ON & ON & ON & OFF & Weibull & 2.34 & 3.60 & 8.69 & 0 \\
ON & ON & ON & OFF & Occurrence & 2.45 & 4.26 & 14.52 & 0.01 \\
ON & ON & ON & ON & Weibull & 5.55 & 8.79 & 49.96 & 0.77 \\
ON & ON & ON & ON & Occurrence & 6.14 & 8.24 & 49.80 \\
\hline
\end{tabular}


scanned samples will extend the interval between two successive observations, which decreases the performance of the positioning algorithms based on time series, for instance, hidden Markov model.

\section{Acknowledgments}

This paper is an extended version of a paper entitled "The Evaluation of WiFi Positioning in a Bluetooth and WiFi Coexistence Environment" presented at the 2nd International Conference on Ubiquitous Positioning, Indoor Navigation, and Location-based Service. This work was a part of the INdoor Outdoor Seamless Navigation for SEnsing Human Behavior (INOSENSE) project, funded by the Academy of Finland. This work is also supported by the Shanghai Science and Technology Committee under Grant 15511105100, 17DZ1100803 and the National Science and Technology Major Project under Grant GFZX0301010708, partly by the National Natural Science Foundation of China under Grant 61573242 and 61402283

\section{About the authors}

Dr. Ling Pei (born 1977, email:ling.pei@sjtu.edu.cn) is an associate professor of Shanghai Jiao Tong University. He received his Master in mechanical engineering from Jiangxi Agricultural University in 2003 and Ph.D. degree in test measurement technology and instruments from the Southeast University, China, in 2007. From 2007 to 2013, he worked in the Navigation and Positioning Department at Finnish Geodetic Institute (FGl) as a specialist research scientist. Since 2013, he has joined the Shanghai Jiao Tong University, where his research interests include indoor/outdoor seamless positioning, sensor fusion, PDR, SLAM, context-aware and hybrid navigation.

\section{Authors' contributions}

$\mathrm{PL}$ conducted the research. CR carried out the fingerprinting studies. $L J$ contributed to the fingerprinting algorithms. CY designed the Bluetooth data collection platform. CL participant the Bluetooth positioning algorithms study. All authors read and approved the final manuscript.

\section{Competeng interests}

The authors declare that they have no competing interests.

\section{Publisher's Note}

Springer Nature remains neutral with regard to jurisdictional claims in published maps and institutional affiliations.

\section{Author details}

'Shanghai Key Laboratory of Navigation and Location-based Services, School of Electronic Information and Electrical Engineering, Shanghai Jiao Tong University, Shanghai, China. ${ }^{2}$ State Key Laboratory of Information Engineering in Surveying, Mapping and Remote Sensing, Wuhan University, Wuhan 430079, China. ${ }^{3}$ Department of Remote Sensing and Photogrammetry, Finnish Geospatial Research Institute, Kirkkonummi, Finland.

Received: 20 June 2016 Accepted: 5 July 2016

Published online: 11 October 2017

\section{References}

Bahl P, Padmanabhan V (2000) N.(2000), RADAR: an in-building RF-based user location and tracking system. Proceedings of IEEE Infocom 2000:775-784

Chen R, Chen Y, Pei L, Chen W, Liu J, Kuusniemi H, Leppäkoski H, Takala J (2009) A DSP-based multi-sensor multi-network positioning platform, In proceedings of the 22nd international technical meeting of the satellite division of the Institute of Navigation (ION GNSS 2009), pp 615-621

Chen W, Chen R, Chen Y, Kuusniemi H, Wang J (2010) An effective pedestrian dead reckoning algorithm using a unified heading error model, in position location and navigation symposium (PLANS), 2010 IEEE/ION. IEEE, USA, pp 340-347

Chen R, Chen W, Chen X, Zhang X, Chen Y (2011a) Sensing strides using EMG signal for pedestrian navigation. GPS Solutions 15(2):161-170

Chen W, Chen R, Chen X, Zhang X, Chen Y, Wang J, Fu Z (2011c) Comparison of EMG-based and accelerometer-based speed estimation methods in pedestrian dead reckoning. J Navig 64(2):265-280

Chen L, Kuusniemi H, Chen Y, Pei L, Kroger T, Chen R (2011 b) Information filter with speed detection for indoor Bluetooth positioning. In localization and GNSS (ICL-GNSS), 2011c, International conference on. IEEE, USA, pp 47-52
Chen L, Kuusniemi H, Chen Y, Pei L, Kröger T, Chen R (2012a) Motion restricted information filter for indoor Bluetooth positioning. Internationa Journal of Embedded and Real-Time Communication Systems (IJERTCS) 3(3):54-66

Chen R, Pei L, Liu J, Leppäkoski H (2012b) WLAN and bluetooth positioning in smart phones, ubiquitous positioning and mobile location-based Services in Smart Phones, pp 44-68

Chen L, Pei L, Kuusniemi H, Chen Y, Kröger T, Chen R (2013) Bayesian fusion for indoor positioning using bluetooth fingerprints. Wirel Pers Commun 1:1-11

Ekahau Inc. (2017) http://www.ekahau.com/. Accessed 30 Aug 2017

Foxlin E (2005) Pedestrian tracking with shoe-mounted inertial sensors, computer graphics and applications. IEEE 25(6):38-46

Hightower J, Want R, Borriello G (2000) SpotON: an indoor 3D location sensing technology based on RF signal strength, UW CSE 00-02-02. University of Washington, Department of Computer Science and Engineering, Seattle, $p 1$

Jiang W, Pei L, Xu C, Chen L, Yu W (2015) Two-stage localization scheme using a small-scale linear microphone Array for indoor environment. J Navig 68(5): 915-936. doi:10.1017/S0373463315000107

Kraemer J, Eisfeller B (2009) A-GNSS, a different approach, inside GNSS, sep/Oct issue, vol 2009, pp 52-61

Kuusniemi H, Liu J, Pei L, Chen Y, Chen L, Chen R (2012) Reliability considerations of multi-sensor multi-network pedestrian navigation, IET Radar. Sonar \& Navigation 6(3):157-164

Liu J (2012) Hybrid positioning with smartphones, ubiquitous positioning and mobile location-based Services in Smart Phones, pp 159-194

Liu J, Chen R, Pei L, Chen W, Tenhunen T, Kuusniemi H, Kroger T, Chen Y (2010) Accelerometer assisted robust wireless signal positioning based on a hidden Markov model, In position location and navigation symposium (PLANS), 2010 IEEE/ION. IEEE, USA, pp 488-497

Liu J, Chen R, Pei L, Guinness R, Kuusniemi H (2012) A hybrid Smartphone indoor positioning solution for mobile LBS. Sensors 12(12):17208-17233

Mathews MB, MacDoran PF, Gold KL (2011) SCP enabled navigation using signals of opportunity in GPS obstructed environments. Navigation 58(2):91-110

Mulloni A, Wagner D, Barakonyi I, Schmalstieg D (2009) Indoor positioning and navigation with camera phones. Pervasive Computing, IEEE 8(2):22-31

Pahlavan K, Akgul FO, Heidari M, Hatami A, Elwell JM, Tingley RD (2006) Indoor geolocation in the absence of direct path, wireless communications. IEEE 13(6):50-58

Pahlavan K, Akgul F, Ye Y, Morgan T, Alizadeh-Shabdiz F, Heidari M, Steger C (2010) Taking positioning indoors Wi-Fi localization and GNSS. Inside GNSS 5(3):40-47

Papoulis A (2002) Probability, random variables and stochastic processes, McGraw-hill education (India) Pvt Itd, vol 2002

Pei L, Chen R, Chen Y, Leppakoski H, Perttula A (2009) Indoor/outdoor seamless positioning technologies integrated on smart phone, in advances in satellite and space communications, 2009, SPACOMM 2009. First international conference on. IEEE, USA, pp 141-145

Pei L, Chen R, Liu J, Chen W, Kuusniemi H, Tenhunen T, Kröger T, Leppäkoski H, Takala J (2010a) Motion recognition assisted indoor wireless navigation on a mobile phone, in proceedings of the 23rd international technical meeting of the satellite division of the Institute of Navigation (ION GNSS 2010), pp 3366-3375

Pei L, Chen R, Liu J, Kuusniemi H, Tenhunen T, Chen Y (2010b) Using inquirybased Bluetooth RSSI probability distributions for indoor positioning. Journal of Global Positioning Systems 9(2):122-130

Pei L, Chen R, Liu J, Tenhunen T, Kuusniemi H, Chen Y (2010c) Inquiry-based bluetooth indoor positioning via rssi probability distributions, In advances in satellite and space communications (SPACOMM), 2010 second international conference on. IEEE, USA, pp 151-156

Pei L, Chen R, Liu J, Kuusniemi H, Chen Y, Tenhunen T (2011) Using motionawareness for the 3D indoor personal navigation on a Smartphone, In proceedings of the 24th international technical meeting of the satellite division of the Institute of Navigation (ION GNSS 2011), pp 2906-2912

Pei L, Liu J, Guinness R, Chen Y, Kroger T, Chen R, Chen L (2012a) The evaluation of WiFi positioning in a Bluetooth and WiFi coexistence environment, In ubiquitous positioning, indoor navigation, and location based service (UPINLBS), 2012. IEEE, USA, pp 1-6

Pei L, Liu J, Guinness R, Chen Y, Kuusniemi H, Chen R (2012b) Using LS-SVM based motion recognition for smartphone indoor wireless positioning. Sensors 12(5):6155-6175

Pei L, Guinness R, Chen R, Liu J, Kuusniemi H, Chen Y, Chen L, Kaistinen J (2013) Human behavior cognition using Smartphone sensors. Sensors 13(2):1402-1424 
Pei L, Zhang M, Zou D, Chen R, Chen Y (2016) A survey of crowd sensing opportunistic signals for indoor localization. Mob Inf Syst, 2016:16, 4041291. doi:10.1155/2016/4041291

Priyantha NB, Chakraborty A, Balakrishnan H (2000) The cricket location-support system, in proceedings of the 6th annual international conference on mobile computing and networking. ACM, New York, pp 32-43

Qian J, Pei L, Ma J, Ying R, Liu P (2015a) Vector graph assisted pedestrian dead reckoning using unconstrained Smartphone. Sensors, (2015) 15(3):5032-5057

Qian J, Pei L, Zou D, Liu P (2015b) Optical flow-based gait modeling algorithm for pedestrian navigation using Smartphone sensors. Sensors Journal, IEEE 15(12):6797-6804. doi:10.1109/JSEN.2015.2464696

Rouzaud D, Skaloud J (2011) Rigorous integration of inertial navigation with optical sensors by dynamic networks. J Navig 58:141-152

Sagias C, Karagiannidis K (2005) Gaussian class multivariate Weibull distributions: theory and applications in fading channels, institute of electrical and electronics engineers. Transactions on Information Theory 51(10):3608-3619 2005

Schilit BN, LaMarca A, Borriello G, Griswold WG, McDonald D, Lazowska E, Balachandran A, Hong Jl, Iverson V (2003) Challenge: ubiquitous locationaware computing and the place lab initiative, In proceedings of the 1st ACM international workshop on wireless mobile applications and services on WLAN hotspots. ACM, New York, pp 29-35

Storms W, Shockley J, Raquet J (2010) Magnetic field navigation in an indoor environment, In ubiquitous positioning indoor navigation and location based service (UPINLBS), 2010. IEEE, USA, pp 1-10

Sugano M, Kawazoe T, Ohta Y, Murata M (2006) Indoor localization system using RSSI measurement of wireless sensor network based on ZigBee standard. Targets 538:050

Syrjärinne J. (2001), Studies of modern techniques for personal positioning Tampere University of Technology

Want R, Hopper A, Falcão V, Gibbons J (1992) The active badge location system. ACM Transactions on Information Systems (TOIS) 10(1):91-102

Zhou H, Zou D, Pei L, Ying R, Liu P, Yu W (2015) StructSLAM: visual SLAM with building structure lines. Vehicular Technology, IEEE Transactions on, (2015) 64(4):1364-1375. doi:10.1109/TVT.2015.2388780

\section{Submit your manuscript to a SpringerOpen ${ }^{\circ}$ journal and benefit from:}

- Convenient online submission

- Rigorous peer review

- Open access: articles freely available online

- High visibility within the field

Retaining the copyright to your article

Submit your next manuscript at $\boldsymbol{s p r i n g e r o p e n . c o m ~}$ 